\title{
Considering bottom curvature in depth-averaged open-channel flow modelling, based on curvilinear coordinates
}

\author{
B. J. Dewals ${ }^{1,2}$, S. Erpicum ${ }^{1} \&$ M. Pirotton ${ }^{1}$ \\ ${ }^{1}$ Department ArGEnCo, University of Liege, Belgium \\ ${ }^{2}$ Fund for Scientific Research F.R.S.-FNRS, Belgium
}

\begin{abstract}
Accurately modelling open-channel flows on strongly vertically curved bottoms, such as for instance over a spillway, is a challenge for any depth-averaged flow model. This type of computation requires the use of axes properly inclined along the mean flow direction in the vertical plane and a modelling of curvature effects. The present generalized model performs such computations by means of curvilinear coordinates in the vertical plane, enabling for instance one to simulate within one single computation domain the flows in the upstream reservoir, over the spillway, in the stilling basin and in the river reach downstream of a dam.

The frame of reference is chosen in such a way that one of the two curvilinear axes follows the local bottom curvature. Hence the set of generalized shallow water equations involves explicitly not only the channel bottom slope, but also the channel vertical curvature and its derivative. The velocity profile is generalized in comparison with the uniform one usually assumed in the conventional shallow-water equations. The pressure distribution is also modified as a function of the bottom curvature and is thus not purely hydrostatic but accounts for effects of centrifugal forces.

This enhanced mathematical modelling framework has been implemented in a 2D finite volume model. A specific flux vector splitting technique has been developed and demonstrated to be stable for any flow regime and any bottom curvature. The scheme offers the advantage of being dependent only on the sign of the bottom curvature. For a vanishing bottom curvature, the new model converges smoothly towards the conventional shallow-water equations.

Finally, two test cases are detailed and lead to satisfactory validation results for the new model.
\end{abstract}

Keywords: shallow-water equations, finite volume, bottom curvature. 


\section{Introduction}

In the conventional shallow-water equations, the main assumption states that velocities normal to a main flow direction are smaller than those in this main flow direction. As a consequence, the pressure field is found to be almost hydrostatic everywhere and a depth-averaging operation of the local conservation laws can be performed easily.

The large majority of flows occurring in rivers can reasonably be seen as shallow and are characterized by relatively small vertical velocity components everywhere, except near local singularities. Those singularities, such as weirs or spillways, can however play a major part in the flow behaviour in the vicinity of the structure, but also in the far field upstream and downstream. A key characteristic of most of those structures is the development of flows over strongly vertically curved bottoms.

While applying fully 3D flow models for large-scale real applications remains challenging in terms of computation cost and data requirements, modelling such flows over vertically curved bottoms is far from straightforward for any depthaveraged model. Indeed, such a computation requires the use of axes properly inclined along the local mean flow direction in the vertical plane. Since this local mean flow direction changes over the computation domain, a suitable modelling of the vertical curvature effects is needed.

Therefore, the present paper describes an extension of the shallow-water equations (SWE), based on a similar depth-averaging operation as in the development of the conventional SWE, but considering a frame of reference with curvilinear lines of coordinates following the main flow direction. As a consequence, the new set of equations includes terms involving not only the channel inclination $\theta[-]$, but also containing explicitly the bottom curvature $\kappa\left[\mathrm{L}^{-1}\right]$ and its rate of change $\kappa^{\prime}\left[\mathrm{L}^{-2}\right]$. The velocity profile is different from the uniform one usually assumed in the conventional shallow water equations. The pressure distribution is also modified to take into centrifugal forces. As a consequence, the mathematical and computational model described hereafter enables to achieve unified 2D modelling of the flows upstream, downstream and over river training structures (e.g. spillway) in one single simulation.

Besides a literature review, the present paper provides a description of the new depth-averaged model, covering the development of the mathematical model and a short overview of its computational implementation. Two validation test cases are also presented, demonstrating that the enhanced model provides more accurate water depth and bottom pressure results than the SWE.

\section{Literature review}

The first attempt to introduce vertical curvature effects in a shallow-water model was performed in a pioneering work by Dressler [13] in 1978. He developed a $1 \mathrm{D}$ model on a similar idea as in the present case, but without a proper depthaveraging operation. His mathematical formulation was thus non-conservative. Later he presented a modified friction term [14], taking into account the uneven 
velocity profile. Experimental validations of this first model were performed by Sivakumaran et al. [21, 22] and later by Ilhan [18]. A 2D generalization, as well as a finite element computer code to solve the model, have been proposed by Berger and Carey $[4,5]$. A finite volume numerical model for solving the 2D extended model, considering curvature along one direction, has been developed and validated by Dewals et al. [9]. Recently, Anh and Hosoda also derived a generalized formulation handling flows over an arbitrary 3D surface [1]. In parallel, 3D models were also applied to spillway overflows (e.g. Bürgisser and Rutschman [6]), while other authors [19, 20] have modelled flows over vertically curved beds by means of vertically averaged and moment equations. However, this later approach presents the drawback of involving 10 equations in $2 \mathrm{D}$, in comparison with 3 equations in the present case.

\section{Model development}

In this section, the mathematical formulation of the model is described. Bed curvature is considered along one direction $(x)$.

\subsection{Curvilinear frame of reference}

The fundamental idea of the extended model is to replace the Cartesian coordinates $(x, z)$ in the vertical plane by curvilinear coordinates $(\xi, \eta)$ selected in order that:

- the curvilinear abscissa $\xi$ follows the local channel bottom;

- the second curvilinear axis $(\eta)$ is normal to the local bed direction;

- the third axis of reference $(y)$ is simply normal to the plane of curvature.

Figure 1 shows the new axes of reference and the main notations used.

In order to allow the evaluation of the space derivatives involved in the curvature expression and those present in the conservation equations, the

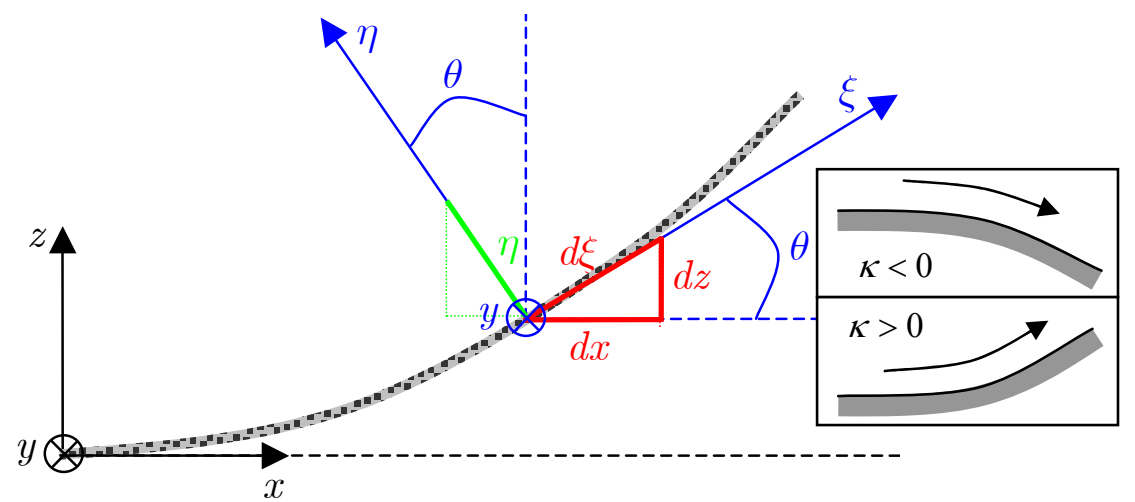

Figure 1: $\quad$ Symbols and axes of reference for the 2D extended model. 
mathematical function describing the channel bed has to be continuous, have a continuous slope $(\theta)$ and a continuous curvature $(\kappa)$. The curve representing the bottom must therefore be three times differentiable.

\subsection{Change of variables}

The correspondence between the Cartesian coordinates and their curvilinear counterparts is given by the following relations:

$$
x=x(\xi, \eta)=x_{\text {bed }}(\xi)-\eta \sin \theta, \quad y=y, \quad z=z(\xi, \eta)=z_{\text {bed }}(\xi)+\eta \cos \theta .
$$

The unit vector tangent to the channel bottom in a vertical plane and the bottom curvature are respectively defined as:

$$
\vec{t}=\left(\begin{array}{l}
d x_{\mathrm{b}} / d \xi \\
d y_{\mathrm{b}} / d \xi \\
d z_{\mathrm{b}} / d \xi
\end{array}\right)=\left(\begin{array}{c}
\cos \theta \\
0 \\
\sin \theta
\end{array}\right) \quad \text { and } \quad \kappa=\left(\vec{t} \wedge \frac{d \vec{t}}{d \xi}\right) \cdot \vec{e}_{y}=\frac{d \theta}{d \xi} .
$$

The Jacobian matrix can be deduced from relation (1), as well as the Jacobian of the change of variables, which is simply equal to the determinant of matrix $\mathrm{J}$ :

$$
\mathrm{J}=\left[\begin{array}{ccc}
\cos \theta(1-\kappa \eta) & 0 & -\sin \theta \\
0 & 1 & 0 \\
\sin \theta(1-\kappa \eta) & 0 & \cos \theta
\end{array}\right], \quad \operatorname{det}(\mathrm{J})=J=1-\kappa \eta
$$

The fundamental relation between derivatives in both systems of reference reads:

$$
\left(\begin{array}{c}
\frac{\partial}{\partial x} \\
\frac{\partial}{\partial y} \\
\frac{\partial}{\partial z}
\end{array}\right)=\left[\begin{array}{ccc}
\cos \theta(1-\kappa \eta)^{-1} & 0 & -\sin \theta \\
0 & 1 & 0 \\
\sin \theta(1-\kappa \eta)^{-1} & 0 & \cos \theta
\end{array}\right]\left(\begin{array}{c}
\frac{\partial}{\partial \xi} \\
\frac{\partial}{\partial y} \\
\frac{\partial}{\partial \eta}
\end{array}\right)
$$

A change of variables is valid provided that the corresponding Jacobian remains positive. In the case of a negative bed curvature (convex), no special restriction needs to be introduced. On the contrary, if the bed curvature is positive (concave), the coordinate $\eta$ must be limited to a value depending on the sharpness of the curvature. The Jacobian expression above remains positive as long as the water height verifies the following condition (with $\kappa>0$ and $R$ the radius of curvature): $H<1 / \kappa=R$.

\subsection{Velocity transformation and velocity profile}

The velocity vector $\vec{v}$ may be expressed in both frames of reference:

$$
\vec{v}=U \vec{e}_{\xi}+V \vec{e}_{y}+W \vec{e}_{\eta}=u \vec{e}_{x}+v \vec{e}_{y}+w \vec{e}_{z} .
$$


Those components are related by the simple transformation formula:

$$
\left(\begin{array}{c}
U \\
V \\
W
\end{array}\right)=\left[\begin{array}{ccc}
\cos \theta & 0 & \sin \theta \\
0 & 1 & 0 \\
-\sin \theta & 0 & \cos \theta
\end{array}\right]\left(\begin{array}{l}
u \\
v \\
w
\end{array}\right) .
$$

Following the work by Dressler [13], the most straightforward assumption concerning velocity distribution has been selected. This corresponds to a nonrotational flow in the vertical plane, expressed in curvilinear coordinates by:

$$
\operatorname{rot} \vec{v} \cdot \vec{e}_{y}=0 \quad \Rightarrow \quad \frac{\partial W}{\partial \xi}=J \frac{\partial U}{\partial \eta}-\kappa U .
$$

Finally, heeding the shallowness of the flow, the corresponding $\xi$-velocity profile is given by: $U(\xi, y, \eta, t)=(1-\kappa \eta)^{-1} C(\xi, y, t)$, with $C$ representing the longitudinal velocity along the channel bottom. The profile of the velocity is hence completely determined by the condition of irrotationality and the bottom curvature, as shown in Figure 2(a).
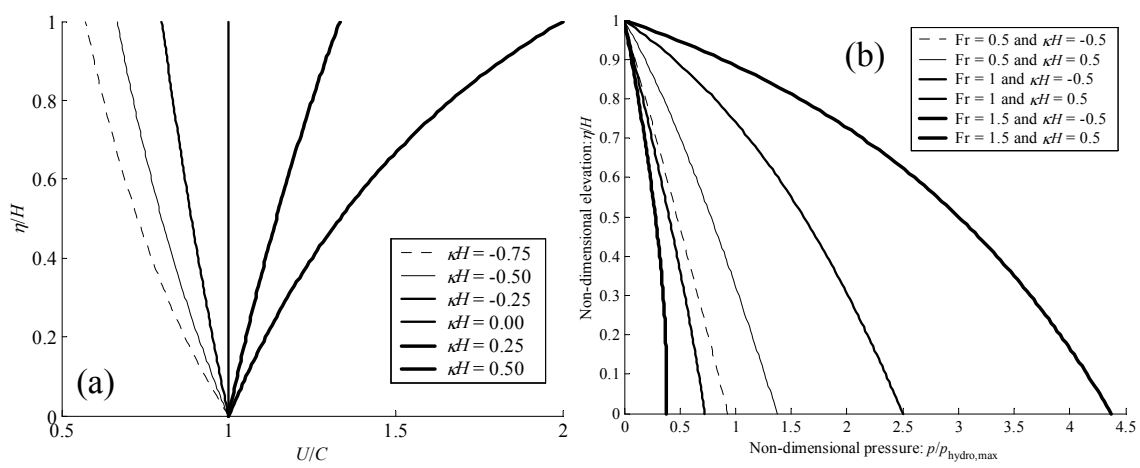

Figure 2: (a) Velocity profiles for different values of the non-dimensional curvature; (b) generalized pressure distributions for various Froude numbers and non-dimensional curvatures $\kappa H$.

\subsection{Local conservation laws for the flow}

A general conservation law, such as

$$
\frac{\partial s}{\partial t}+\frac{\partial f_{x}}{\partial x}+\frac{\partial f_{y}}{\partial y}+\frac{\partial f_{z}}{\partial z}=S
$$

may be transformed in the system of curvilinear coordinates by applying the fundamental relation (4). This operation leads to a new conservative law:

$$
\frac{\partial(J s)}{\partial t}+\frac{\partial}{\partial \xi}\left(f_{x} \cos \theta+f_{z} \sin \theta\right)+\frac{\partial\left(J f_{y}\right)}{\partial y}+\frac{\partial}{\partial \eta}\left(f_{z} J \cos \theta-f_{x} J \sin \theta\right)=J S,
$$


which can later on be used to transform the mass and momentum conservation equations for the flow. The local continuity equation can be easily deduced from its Cartesian form and the general relation (9):

$$
\frac{\partial U}{\partial \xi}+\frac{\partial}{\partial y}(J V)+\frac{\partial}{\partial \eta}(J W)=0 .
$$

The same transformation performed for the momentum conservation equations leads to three new equations, which can be combined to provide the local momentum conservation laws expressed in curvilinear coordinates:

$$
\begin{gathered}
\frac{\partial}{\partial t}(J U)+\frac{\partial}{\partial \xi}\left(U^{2}+p / \rho\right)+\frac{\partial}{\partial y}(J U V)+\frac{\partial}{\partial \eta}(J U W)=-g J \sin \theta+\kappa U W, \\
\frac{\partial}{\partial t}(J V)+\frac{\partial}{\partial \xi}(U V)+\frac{\partial}{\partial y}\left[J\left(V^{2}+p / \rho\right)\right]+\frac{\partial}{\partial \eta}(J V W)=0 \\
\frac{\partial(J W)}{\partial t}+\frac{\partial(U W)}{\partial \xi}+\frac{\partial(J V W)}{\partial y}+\frac{\partial}{\partial \eta}\left[J\left(W^{2}+\frac{p}{\rho}\right)\right]=-g J \cos \theta-\kappa\left(U^{2}+\frac{p}{\rho}\right) .
\end{gathered}
$$

According to the conventional assumption regarding the shallowness of the flow ( $W^{2} \ll U^{2}+V^{2}$ ), the purely advective terms expressing an acceleration perpendicular to the bottom may be ignored in the latter equation. A complete non-dimensional analysis of orders of magnitude has been performed and demonstrates that this conclusion remains valid for the model expressed in curvilinear coordinates. As a result, the generalized pressure distribution can be deduced directly by integrating relation (13) along the $\eta$-axis:

$$
p(\eta) / \rho=g \cos \theta(H-\eta)+C^{2} / 2\left[(1-\kappa H)^{-2}-(1-\kappa \eta)^{-2}\right] .
$$

It can be easily verified that in the case of a zero-curvature bed, the term in square brackets vanishes in relation (14) and hence the hydrostatic pressure distribution is restored. Figure 2(b) illustrates the effect of bed curvature on the pressure field as a function of a modified Froude number, defined as $\operatorname{Fr}=C / \sqrt{g H \cos \theta}$. The figure highlights a higher sensitivity of the pressure profile to positive curvatures, rather than to negative ones.

\subsection{Boundary conditions}

The interface between the studied liquid and the ambient air is defined by the impossibility for the fluid to cross this surface. This implies that the velocity of the fluid in the direction normal to the interface and the velocity of this interface in the same normal direction are equal. According to Whitham [23], and taking into consideration the specific velocity profile of the flow over a vertically curved bed, this equality of normal velocities can be expressed as follows:

$$
W(t, \xi, y, H)=\frac{\partial H}{\partial t}+\frac{U(t, \xi, y, H)}{1-\kappa H} \frac{\partial H}{\partial \xi}+V(t, \xi, y, H) \frac{\partial H}{\partial y} .
$$

The no-flow condition across the bottom is expressed by: $W(t, \xi, y, 0)=0$. 
Since the free surface position is not known a priori, its location constitutes an additional unknown and a second boundary condition is required. This dynamic boundary condition simply states that the pressure at the free surface is identical to the atmospheric pressure, selected as the reference: $p(\xi, y, H, t)=p_{\text {atm }}=0$.

\subsection{Depth-averaged equations for the flow}

Integrating equation (10) from the bottom $(\eta=0)$ to the free surface $(\eta=H)$ leads to the depth-averaged mass conservation equation:

$$
\frac{\partial \mathcal{H}}{\partial t}+\frac{\partial q}{\partial \xi}+\frac{\partial}{\partial y}(\mathcal{H} V)=0
$$

where $\mathcal{H}$ and $q$ represent respectively the actual "volume" in a cell and the unit discharge, defined as:

$$
\mathcal{H} \triangleq H \bar{J}=H\left(1-\frac{\kappa H}{2}\right) \quad \text { and } \quad q(\xi, t)=H \bar{U}=\int_{0}^{H(\xi, t)} U(\xi, \eta, t) d \eta .
$$

The previous expression can be evaluated for the particular velocity profile:

$$
q=H C(-\kappa H)^{-1} \ln (1-\kappa H) .
$$

A similar procedure applied to equations (11) and (12) leads to the momentum conservation along the $\xi$-axis:

$$
\begin{array}{r}
\frac{\partial}{\partial t}(H C)+\frac{\partial}{\partial \xi}\left(\frac{H C^{2}}{1-\kappa H}\right)+\frac{\partial}{\partial y}(H C V)+\frac{\partial}{\partial \xi}\left[g \frac{H^{2}}{2} \cos \theta+\frac{H C^{2}}{2} \frac{\kappa H}{(1-\kappa H)^{2}}\right] \\
=-g \mathcal{H} \sin \theta+\kappa H \overline{U W}
\end{array}
$$

and the corresponding relation along the $y$-axis:

$$
\frac{\partial}{\partial t}(\mathcal{H} V)+\frac{\partial}{\partial \xi}(V q)+\frac{\partial}{\partial y}\left(\mathcal{H} V^{2}+H \bar{J} \frac{p}{\rho}\right)=0,
$$

with the mean pressure term written out in full as follows:

$$
H J \frac{\bar{p}}{\rho}=g \frac{H^{2}}{2} \cos \theta\left(1-\frac{\kappa H}{3}\right)+\frac{H C^{2}}{2} \frac{1-\kappa H / 2}{(1-\kappa H)^{2}}-\frac{H C^{2}}{2} \frac{\ln (1-\kappa H)}{-\kappa H} .
$$

It can be easily verified that in the case of a zero curvature bed, equations (16), (19) and (20) become identical to the set of the conventional shallow-water equations. According to the non-dimensional analysis of orders of magnitude, the term $\kappa H \overline{U W}$ in (19) may not necessarily be ignored. It can be accounted for thanks to a proper assumption regarding the profile of the velocity component $W$ (normal to the bottom), as detailed for instance by Dewals et al. [9].

A bottom friction term can be added on the right hand side of equations (20) and (21). Dressler and Yevjevich [14] suggest an adapted formulation of the conventional empirical friction laws (Chezy, Manning) for a 1D model including bed curvature. In addition, in further developments of the present research, the 
velocity profile will be enhanced to take into account the corresponding bottom shear stress.

\section{Computational model}

The system of equations (16), (19) and (20) remains conservative, which is a crucial asset for numerical discretization. In the present case, a finite volume scheme is used for the space discretization of the system. An original Flux Vector Splitting (FVS) is used for the space discretization of the complete set of equations [7]. This second order upwind scheme is Froude independent and depends only on the sign of the bottom curvature. Its stability has been demonstrated by means of a theoretical study of the mathematical system [7,9], as well as a von Neumann stability analysis [7]. The computational model deals with multiblock Cartesian grids. The multiblock organization of the computation code enables the activation of the extended model only in the vicinity of the spillway, while the simple SWE are solved in the rest of the computation domain. This feature leads to CPU time savings.

Several expressions in the additional terms of the extended model present a very stiff behaviour for low-value curvatures. For this reason, expressions, such as the specific discharge (18) or the mean pressure term (21), are replaced in the numerical model by a series expansion as soon as $\kappa H$ becomes very small.

The herein described model is integrated within the modelling system WOLF, developed at the University of Liege. This set of interconnected computational models enables the simulation of process-oriented hydrology [3], 1D [3, 15] and $2 \mathrm{D}$ hydrodynamics $[2,16,17]$, sediment or pollutant transport $[7,10,11]$, air entrainment [8], turbulence (incl. a $k-\varepsilon$ closure) [15] ... as well as optimisation processes (based on Genetic Algorithms) [15]. Other functionalities of WOLF include the use of moment of momentum equations [7] or the analysis of flow instabilities [12].

\section{Validation}

This section aims at demonstrating the improvements brought by the generalized SWE model in terms of results accuracy and realism. The section includes comparisons between the SWE and the extended model for idealized test cases. Full details can be found in the original reference cited for each example.

\subsection{Symmetric profile}

Sivakumaran et al. [22] studied the flow over a symmetric profile in a flume of $9.15 \times 0.75 \times 0.445 \mathrm{~m}$. The flume roughness corresponds to a Manning coefficient $n \approx 0.01 \mathrm{~m}^{-1 / 3} \mathrm{~s}$ and experiments were carried out with two different discharges: $0.11197 \mathrm{~m}^{2} / \mathrm{s}$ and $0.03599 \mathrm{~m}^{2} / \mathrm{s}$.

In both cases, the present extended model with curvature leads to significantly more accurate predictions for the free surface location, as well as for the bed pressure. Results for the higher discharge are presented in Figures 3(a) and (b). 

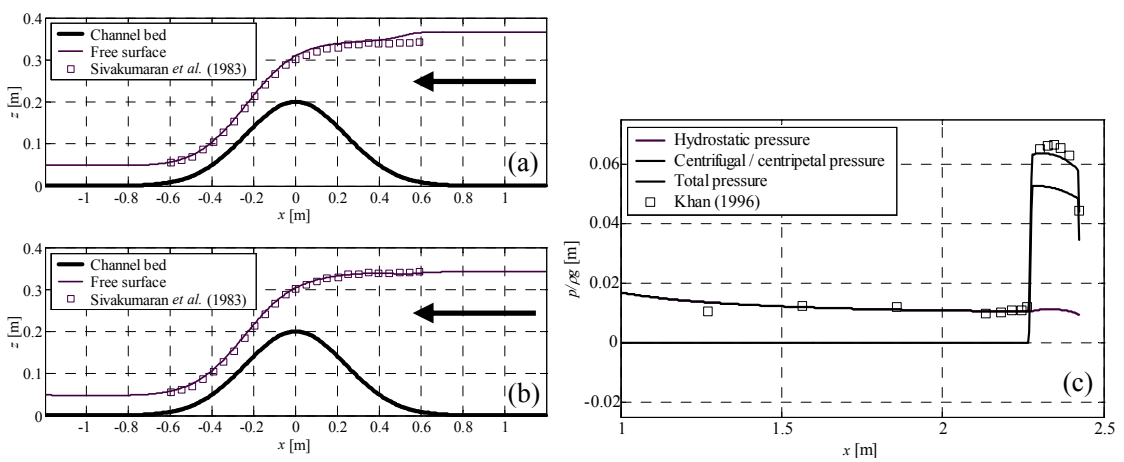

Figure 3: $\quad$ Water surface for the symmetric profile of Sivakumaran et al. [22], simulated with the SWE (a) and with the extended model (b); bed pressure on the flip-bucket spillway tested by Khan and Steffler [19] (c).

\subsection{Flip-bucket spillway}

Water profile and bed pressure measurements, conducted on a laboratory flipbucket spillway, were presented by Khan and Steffler [19] in 1996. The slope of the chute was $20^{\circ}$ and the idealized flip-bucket was represented by a circular arc of radius $15.4 \mathrm{~cm}$. Two specific discharges were tested: $0.0187 \mathrm{~m}^{2} / \mathrm{s}$ and $0.0292 \mathrm{~m}^{2} / \mathrm{s}$. Figure 3(c) shows a comparison between observed and computed pressures on the bottom of the spillway for the higher discharge. The pressure profile computed with the herein presented model agrees well with the measurements.

\section{Conclusion}

Since the shallow-water equations simply assume that both the bed curvature and its rate of change may be neglected, such an approach is valid only if no sharp changes in the bed inclination occur. Though these conditions are very widely met in waterways and rivers, experience in open channel flow modelling demonstrates the necessity for a better approximation for a wide range of applications, such as spillway overflows. Therefore, the herein presented 2D generalized model has been developed in order to handle correctly the effects induced by strong bottom curvatures, based on curvilinear coordinates in the vertical plane. The model presented generalizes the shallow water theory by incorporating effects of streamline vertical curvature. It leads to better estimations of particle velocity profiles and non-hydrostatic pressure distribution over curved surfaces. More accurate upstream water elevation and bottom pressure can thus be predicted.

Moreover, the approach enables simulations completely integrating the flow behaviour in the vicinity of large river structures, such as dams: the flows in the 
upstream reservoir, on the spillway, in the stilling basin and in the downstream river reach can be treated in one single simulation.

The full development of the mathematical model has been presented and two successful test-cases have been described. Both cases lead to significant improvements in the numerical results compared to the conventional SWE.

\section{References}

[1] Anh, T.N. and T. Hosoda, Depth-averaged model of open-channel flows over an arbitrary 3D surface and its applications to analysis of water surface profile. J. Hydraul. Eng.-ASCE, 2007. 133(4): pp. 350-360.

[2] Archambeau, P., B. Dewals, S. Detrembleur, S. Erpicum and M. Pirotton, A set of efficient numerical tools for floodplain modeling, in Shallow Flows, G.H. Jirka and W.S.J. Uijttewaal (eds). 2004, Balkema: Leiden, etc. pp. 549-557.

[3] Archambeau, P., Contribution à la modélisation de la genèse et de la propagation des crues et inondations. 2006, $\mathrm{PhD}$ thesis, University of Liege.

[4] Berger, R.C. and G.F. Carey, Free-surface flow over curved surfaces - Part I: perturbation analysis. Int. J. Numer. Meth. Fluids, 1998. 28(2): pp. 191200.

[5] Berger, R.C. and G.F. Carey, Free-surface flow over curved surfaces - Part II: computational model. Int. J. Numer. Meth. Fluids, 1998. 28(2): pp. 201213.

[6] Bürgisser, M.F. and P. Rutschmann. Numerical solution of viscous 2DV free surface flows: flow over spillway crests. in 28th IAHR Congress Graz. 1999.

[7] Dewals, B., Une approche unifiée pour la modélisation d'écoulements à surface libre, de leur effet érosif sur une structure et de leur interaction avec divers constituants. 2006, PhD thesis, University of Liege: 636 pp.

[8] Dewals, B.J., S. André, A. Schleiss and M. Pirotton. Validation of a quasi2D model for aerated flows over stepped spillways for mild and steep slopes. in Proc. 6th Int. Conf. of Hydroinformatics. 2004. Singapore.

[9] Dewals, B.J., S. Erpicum, P. Archambeau, S. Detrembleur and M. Pirotton, Depth-integrated flow modelling taking into account bottom curvature. J. Hydraul. Res., 2006. 44(6): pp. 787-795.

[10] Dewals, B.J., S. Erpicum, P. Archambeau, S. Detrembleur and M. Pirotton. Hétérogénéite des échèlles spatio-temporelles d'écoulements hydrosédimentaires et modélisation numerique. in Transports solides et gestion des sédiments en milieux naturels et urbains. 2007. Lyon, France: SHF.

[11] Dewals, B.J., P. Archambeau, S. Erpicum and M. Pirotton, A modelling system for open-channel flows with mobile bed - Application as a decisionsupport tool for sediment management in a hydropower project. Geophysical Research Abstracts, 2008. 10. 
[12] Dewals, B.J., S.A. Kantoush, S. Erpicum, M. Pirotton and A.J. Schleiss, Experimental and numerical analysis of flow instabilities in rectangular shallow basins. Environ. Fluid Mech., 2008 (accepted).

[13] Dressler, R.F., New Nonlinear Shallow Flow Equations with Curvature. J. Hydraul. Res., 1978. 16(3): pp. 205-222.

[14] Dressler, R.F. and V. Yevjevich, Hydraulic-resistance terms modified for the Dressler curved-flow equations. J. Hydraul. Res., 1984. 22(3): pp. 145156.

[15] Erpicum, S., Optimisation objective de paramètres en écoulements turbulents à surface libre sur maillage multibloc. 2006, PhD thesis, University of Liege.

[16] Erpicum, S., P. Archambeau, S. Detrembleur, B. Dewals and M. Pirotton, A 2D finite volume multiblock flow solver applied to flood extension forecasting, in Numerical modelling of hydrodynamics for water ressources, P. García-Navarro and E. Playán (eds). 2007, Taylor \& Francis: Londres. pp. 321-325.

[17] Erpicum, S., B.J. Dewals, P. Archambeau, S. Detrembleur and M. Pirotton. Detailed 2D numerical modeling for flood extension forecasting. in Proc. 4th Int. Conf. on Fluvial Hydraulics: River Flow 2008. 2008 (accepted).

[18] Ilhan, M.H., Computation of flow over vertically curved channels, in Gradual School of Natural and Applied Sciences. 1994, Master thesis, Middle East Technical University, Ankara.

[19] Khan, A.A. and P.M. Steffler, Vertically averaged and moment equations model for flow over curved beds. J. Hydraul. Eng.-ASCE, 1996. 122(1): pp. 3-9.

[20] Khan, A.A. and P.M. Steffler, Modelling overfalls using vertically averaged and moment equations. J. Hydraul. Eng.-ASCE, 1996. 122(7): pp. 397-402.

[21] Sivakumaran, N.S., R.J. Hosking and T. Tingsanchali, Steady shallow flow over a spillway. J. Fluid Mech., 1981. 111: pp. 411-420.

[22] Sivakumaran, N.S., T. Tingsanchali and R.J. Hosking, Steady shallow flow over curved beds. J. Fluid Mech., 1983. 128: pp. 469-487.

[23] Whitham, G.B., Linear and Nonlinear Waves. 1974, New York: Wiley, 636 pp. 